\title{
A Catalog of Active Galactic Nuclei from the First 1.5 Gyr of the Universe
}

\author{
Krisztina Perger $^{1 *}$, Sándor Frey ${ }^{2}$, Krisztina É. Gabányi ${ }^{2}$ and L. Viktor Tóth ${ }^{1}$ \\ ${ }^{1}$ Department of Astronomy, Eötvös Loránd University, Budapest, Hungary, ${ }^{2}$ Konkoly Observatory, MTA Research Centre for \\ Astronomy and Earth Sciences, Budapest, Hungary
}

\section{OPEN ACCESS}

Edited by:

Mauro D'Onofrio,

University of Padua, Italy

Reviewed by:

Giovanna Maria Stirpe,

Osservatorio Astronomico di Bologna

(INAF), Italy

Daniela Bettoni,

Osservatorio Astronomico di Padova

(INAF), Italy

Alenka Negrete,

National Autonomous University of

Mexico, Mexico

*Correspondence:

Krisztina Perge

k.perger@astro.elte.hu

Specialty section:

This article was submitted to

Milky Way and Galaxies,

a section of the journal

Frontiers in Astronomy and Space

Sciences

Received: 22 June 2017

Accepted: 21 July 2017

Published: 08 August 2017

Citation:

Perger K, Frey S, Gabányi KÉ and Tóth LV (2017) A Catalog of Active Galactic Nuclei from the First 1.5 Gyr

of the Universe.

Front. Astron. Space Sci. 4:9. doi: 10.3389/fspas.2017.00009
Active galactic nuclei (AGN) are prominent astrophysical objects that can be observed throughout the whole Universe. To understand the underlying physical processes and the different appearance of AGN types, extensive samples are needed. Nowadays, various AGN catalogs are available at different wavebands. However, at the highest redshifts data are still relatively sparse. These data are required for examining AGN properties in the early Universe. This way we can compare the earliest AGN with those seen at lower redshifts, and can study their cosmological evolution. Additionally, because of their high luminosity, AGN may also be used as probes to test cosmological models. With the aim of constructing a complete sample of all known $A G N$ at $z \geq 4$, we are currently compiling a photometric catalog from literature sources. We cross-match catalogs particularly at optical and radio wavebands, to build up a sample for detailed high-resolution radio interferometric studies. The continuously updated list now contains nearly 2,600 objects with known spectroscopic redshifts, optical magnitudes, and auxiliary information about observations at other wavebands. About 170 of them are known radio sources for which we collect existing radio interferometric data from the literature.

Keywords: quasars, active galactic nuclei, extragalactic astronomy, photometry, spectroscopy, catalogs

\section{INTRODUCTION}

Active galactic nuclei (AGN) have been extensively studied for more than half a century. The activity of AGN is believed to originate from material falling into an accreting supermassive black hole (SMBH) in the central region of the galaxy, as first proposed by Lynden-Bell (1969). Due to the accretion process, enormous amount of energy is released, which can be observed throughout the entire electromagnetic spectrum (e.g., Urry and Padovani, 1995). In the cases of radio-loud (jetted) AGN, strong radio emission is produced via synchrotron emission in a symmetric pair of jets emanating from the vicinity of the central SMBH (Blandford and Rees, 1974; Blandford and Königl, 1979). However, the formation and physical properties of AGN are not yet fully understood (e.g., Netzer, 2015).

The large variety of observed characteristics of AGN require sophisticated models to provide an adequate description of the underlying physics. To determine their properties satisfactorily, we need both low- and high-redshift populations of AGN, which requires statistically complete samples.

To date, several different AGN catalogs and samples exist at various wavebands (e.g., Constantin et al., 2009; Véron-Cetty and Véron, 2010; Shen et al., 2011; Kalfountzou et al., 2014; Secrest et al., 2015). We are currently compiling a catalog using extensive samples of AGN primarily discovered by optical observations in recent years, with the aim of constructing a complete sample of AGN at the earliest epochs of the Universe. Given the accelerating pace of data acquisition of 


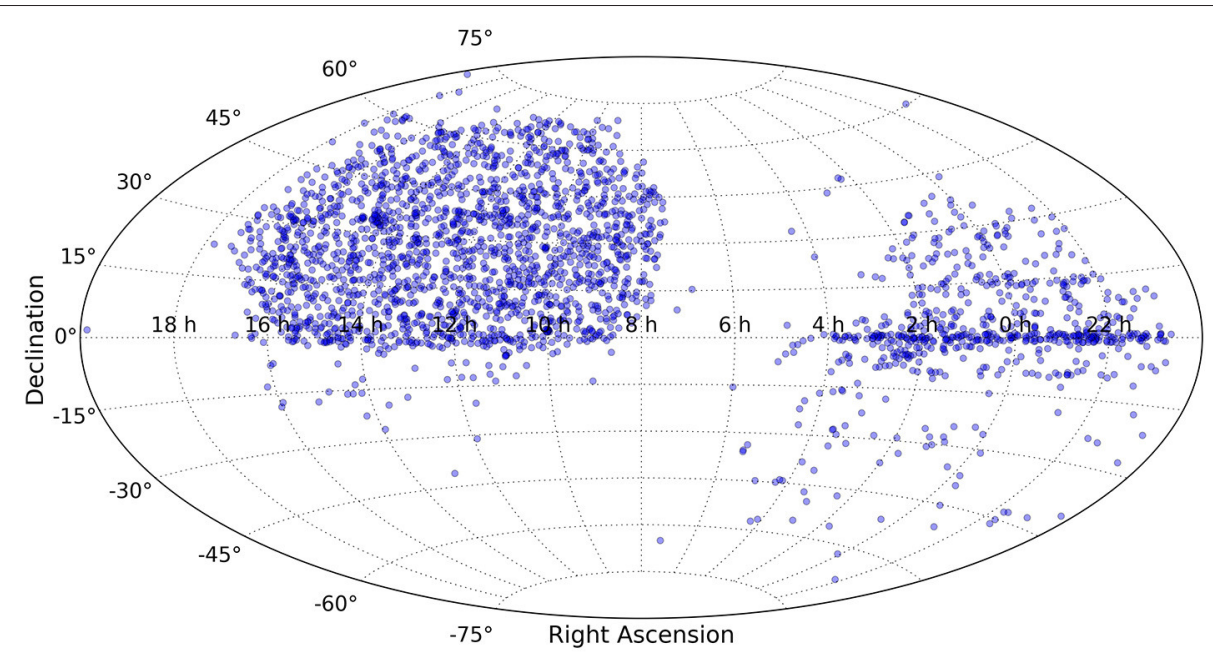

FIGURE 1 | Sky coverage of the sources at the current state of the catalog.

contemporary optical sky surveys, we intend to update and extend our catalog regularly.

To better understand the observable characteristics of AGN, we collected available data at radio wavebands as well, including flux densities from large radio surveys and interferometric observations of individual objects. Our catalog may facilitate several scientific studies. The future applications carry the opportunity to determine the luminosity function of AGN at the highest redshifts (e.g., Fan et al., 2004; Willott et al., 2010; Jiang et al., 2016). It can also provide sample for studies of galaxy environment and possible overdensities around high-redshift quasars (e.g., Orsi et al., 2016; Uchiyama et al., 2017). With supplementary UV data, it may become useful for cosmological studies, providing a sample where 'standard candles' can be selected from (e.g., Marziani and Sulentic, 2014). Likewise, using a sample of the most distant compact radio-emitting AGN, further constraints on the angular size-redshift relation could be obtained (e.g., Gurvits et al., 1999). Presumably, it will make the planning of interferometric observations for sufficiently bright radio sources simpler. Furthermore, it might aid in the selection of interesting individual objects for detailed analysis, and in examining the Galactic foreground in different wavebands.

To compile our sample of high-redshift AGN, we applied a somewhat arbitrary $z \geq 4$ threshold for redshifts. In this regime, the first AGN (QSO 0046-293) was discovered by Warren et al. (1987). Since then, the known number of sources at the earliest epochs of the Universe increased at a fast pace, primarily due to extensive all-sky surveys, e.g., the Sloan Digital Sky Survey (SDSS) (York et al., 2000; Eisenstein et al., 2011; Alam et al., 2015).

\section{COMPILING THE CATALOG}

We built the base of the catalog using 3 large samples of AGN: the quasars of the twelfth data release of SDSS (Jiang et al., 2016; Pâris et al., 2017), the high-redshift quasar sample (Bañados et al., 2016, and references therein) of the Panoramic Survey Telescope and Rapid Response System (Pan-STARRS, Chambers et al., 2016) Survey and the Half Million Quasars (HMQ) Catalog (Flesch, 2015). We filtered the HMQ and SDSS quasars for redshifts $z \geq 4$. Since sources in the Pan-STARRS high-redshift quasar sample had redshifts $z \geq 5.6$, no filtering was needed.

The bulk of the catalog was then constructed after crossmatching the three large samples above. First, HMQ and SDSS quasars were matched. To avoid duplicate entries, we were looking for SDSS-discovered objects in the HMQ sample. Based on the results of e.g., Barro et al. (2011) and Rimoldini et al. (2012), we applied a search radius of $1^{\prime \prime}$ and considered the objects within this radius identical if found in both catalogs. There were 2,363 objects in the HMQ catalog and 1,881 in the SDSS quasar sample at $z \geq 4$. All but 2 SDSS sources were also included in the HMQ sample. The match therefore resulted in 2,365 independent sources. Then the HMQ-SDSS sample was cross-matched with the Pan-STARRS quasar list, applying the same search radius of $1^{\prime \prime}$. This increased the number of unique sources to 2,470 . The cross-matching and the concatenation of independent source lists were carried out using the topcat catalog editing software ${ }^{1}$ (Taylor, 2005).

The resulting catalog contained the following properties: right ascension and declination (optical) coordinates, name, radio identification and X-ray identification names (when existing), redshift, optical magnitudes if known, and literature references regarding discovery and redshift data.

In the course of catalog updates, we added 121 new sources to the list. These were found in the literature (McMahon et al., 2002; Richards et al., 2009; Yi et al., 2014; Wang et al., 2016; Khorunzhev et al., 2017; Matsuoka et al., 2017; Reed et al., 2017; Tang et al., 2017; Tuccillo et al., 2017; Wang et al., 2017; Yang et al., 2017; Yi et al., 2017) but not contained in the 3 large catalogs

${ }^{1} \mathrm{http}: / /$ www.starlink.ac.uk/topcat/ 


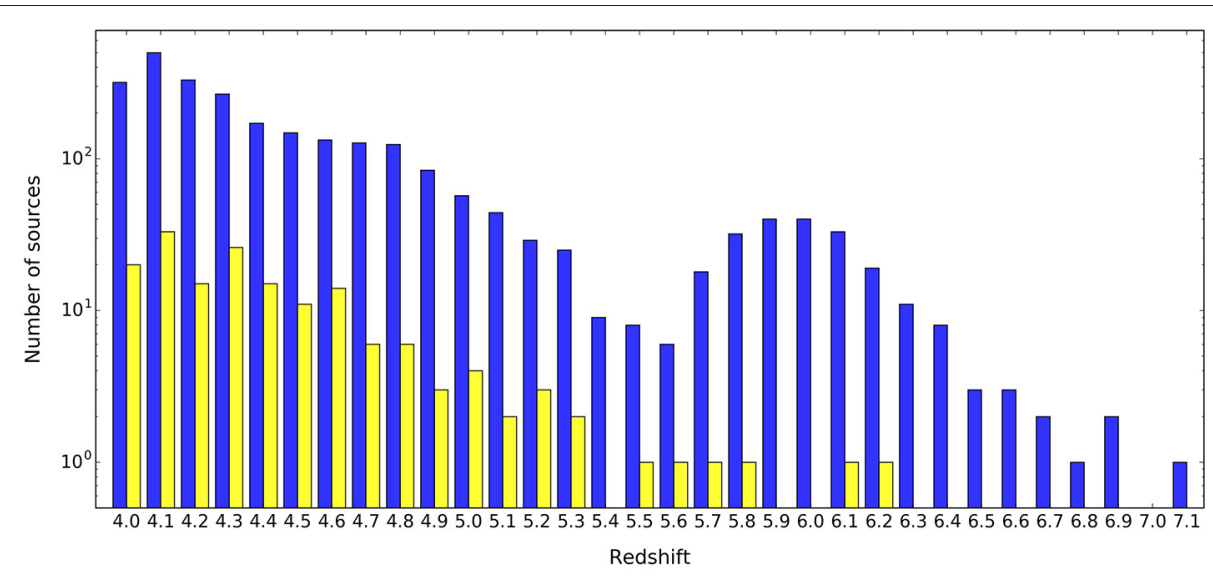

FIGURE 2 | Histogram of the redshift distribution of catalog entries, with a bin width of 0.1. Blue columns indicate all AGN in the sample, while yellow columns show the distribution of sources with available radio flux densities. From the total of 2,591 sources, 166 are detected in FIRST or (if out of FIRST coverage) in NVSS. Two thousand one hundred and forty-eight positions are covered by FIRST but radio sources not detected (brightness upper limit is available only). Two hundred and fifty two sources are outside the FIRST field and lack detection in NVSS, and 25 are outside the footprints of both surveys.

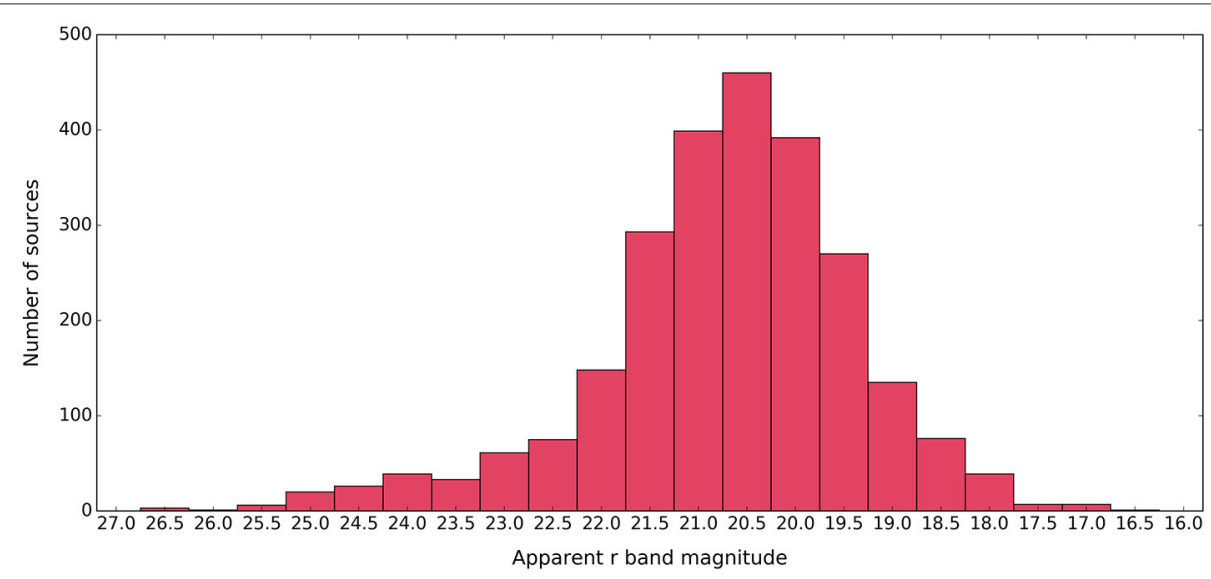

FIGURE 3 | Apparent red magnitude distribution of the high-redshift AGN. The histogram shows the 2,493 sources with available red magnitudes with a bin width of $0.5^{\mathrm{m}}$. Note that since most sources have photometry based on POSS plates, magnitudes might differ from SDSS magnitudes, up to $2^{\mathrm{m}}-3^{\mathrm{m}}$.

that form the basis of our list. The sky coverage of the full sample is plotted in Figure 1.

To find radio counterparts, we searched for the catalog sources in the VLA Faint Images of the Radio Sky at Twenty-Centimeters (FIRST) Survey ${ }^{2}$ (White et al., 1997) database with a $1.5^{\prime \prime}$ radius, the same value that was applied by Ivezić et al. (2002). This resulted in 144 matches. We also marked those 2,148 positions which were covered by FIRST but there were no sources found above the detection limit $(\sim 1 \mathrm{mJy})$, and those which were close to the field edge or lay outside FIRST coverage. Those which were outside the FIRST footprint, were also searched in the NRAO VLA Sky Survey (NVSS) catalog ${ }^{3}$ (Condon et al., 1998), with a search radius of $10^{\prime \prime}$ (Sadler et al., 2002). Positions outside the NVSS field or both survey footprints were also noted. Altogether 166 sources have radio flux densities measured in

\footnotetext{
${ }^{2}$ http://sundog.stsci.edu

${ }^{3}$ http://www.cv.nrao.edu/nvss/
}

either FIRST or NVSS, both at $1.4 \mathrm{GHz}$. This is $6.5 \%$ of the 2,566 sources which were covered by either of the surveys. The redshift distribution of the full sample and of those sources with radio detection are plotted in Figure 2. The prominent "gap" visible between redshifts 5.3 and 5.6 and the excess in the 5.7-6.4 range arises mainly from the sample selection: the Pan-STARRS sample contained only quasars with $z \geq 5.6$. Moreover, the Pan-STARRS survey has a more extensive sky coverage than SDSS: the majority of independent Pan-STARRS quasars have redshifts between 5.7 and 6.4 .

We added very long baseline interferometry (VLBI) flux densities for a total of 35 quasars (Paragi et al., 1999; Beasley et al., 2002; Frey et al., 2003, 2005, 2008, 2010, 2011; Fey et al., 2004; Momjian et al., 2004, 2005, 2008; Romani et al., 2004; Petrov et al., 2006, 2008; Helmboldt et al., 2007; Veres et al., 2010; Cheung et al., 2012; Frey et al., 2013; Cao et al., 2014, 2017; Parijskij et al., 2014; Frey et al., 2015; Gabányi et al., 2015; Coppejans et al., 2016; Wang et al., 2017; Zhang et al., 2017). We also marked sources 
that have already been proposed for VLBI observations to our knowledge.

From the total of 2,591 AGN in the current version of our catalog, we have available red band optical magnitudes for 2,493 quasars. To illustrate this, the distribution of the $r$ band magnitudes of these sources are shown in Figure 3. A descending trend can be seen at the faint end of the histogram due the selection effect casued by the limited sensitivity of the optical surveys. The histogram also shows that there are a couple of particularly bright sources in the sample. Given the high redshifts, these magnitudes would imply extremely high luminosities for these AGN. We therefore investigated this peculiarity by looking at individual objects in our catalog. We found that in some cases sources from the HMQ catalog where the photometry was based on Palomar Observatory Sky Survey (POSS) plates show significant difference from the values observed in SDSS, reaching up to $2^{\mathrm{m}}-3^{\mathrm{m}}$. However, the original HMQ catalog contains a flag indicating the quality of the photometry. We plan to improve our catalog and make sure that only high-quality photometric data are listed in the next version.

\section{SUMMARY}

With the aim of creating a catalog which can be applied to a wide range of studies, we cross-matched and concatenated 3 large lists of AGN, taken from the SDSS twelfth data release, the PanSTARRS first data release, and the HMQ catalog. We expanded this base catalog with numerous sources from the literature. The sample counts 2,591 sources at its current state, from which 166 AGN have available measured radio flux densities. Furthermore, 35 of them already have high-resolution VLBI imaging observations published in the literature. The current version of the catalog can be obtained on request by contacting the corresponding author. We intend to make the $z \geq 4$ AGN catalog publicly available in the near future.

\section{REFERENCES}

Alam, S., Albareti, F. D., Allende Prieto, C., Anders, F., Anderson, S. F., Anderton, T., et al. (2015). The eleventh and twelfth data releases of the Sloan Digital Sky Survey: final data from SDSS-III. Astrophys. J. 219:12. doi: 10.1088/0067-0049/219/1/12

Bañados, E., Venemans, B. P., Decarli, R., Farina, E. P., Mazzucchelli, C., Walter, F., et al. (2016). The Pan-STARRS1 distant $z>5.6$ quasar survey: more than 100 quasars within the first Gyr of the Universe. Astrophys. J. 227:11. doi: 10.3847/0067-0049/227/1/11

Barro, G., Pérez-Gonz ález, P. G., Gallego, J., Ashby, M. L. N., Kajisawa, M., Miyazaki, S., et al. (2011). UV-to-FIR analysis of Spitzer/IRAC sources in the extended groth strip. I. Multi-wavelength photometry and spectral energy distributions. Astrophys. J. 193:13. doi: 10.1088/0067-0049/193/1/13

Beasley, A. J., Gordon, D., Peck, A. B., Petrov, L., MacMillan, D. S., Fomalont, E. B., et al. (2002). The VLBA Calibrator Survey-VCS1. Astrophys. J. 141:13. doi: $10.1086 / 339806$

Blandford, R. D., and Königl, A. (1979). Relativistic jets as compact radio sources. Astrophys. J. 232:34. doi: 10.1086/157262

Blandford, R. D., and Rees, M. J. (1974). A “twin-exhaust” model for double radio sources. Mon. Not. Roy. Astron. Soc. 169:395. doi: 10.1093/mnras/169.3.395

\section{AUTHOR CONTRIBUTIONS}

KP compiled the catalog. All authors participated in defining the catalog content and the preparation of the manuscript.

\section{FUNDING}

This research was partly supported by the grants OTKA NN110333 and NN111016 received from the Hungarian National Research, Development and Innovation Office.

\section{ACKNOWLEDGMENTS}

KP thanks the organizers of the "Quasars at All Cosmic Epochs" conference for support. Funding for SDSS-III has been provided by the Alfred P. Sloan Foundation, the Participating Institutions, the National Science Foundation, and the U.S. Department of Energy Office of Science. The SDSS-III web site is http://www. sdss3.org/. The Pan-STARRS1 Surveys (PS1) and the PS1 public science archive have been made possible through contributions by the Institute for Astronomy, the University of Hawaii, the Pan-STARRS Project Office, the Max-Planck Society and its participating institutes, the Max Planck Institute for Astronomy, Heidelberg and the Max Planck Institute for Extraterrestrial Physics, Garching, The Johns Hopkins University, Durham University, the University of Edinburgh, the Queen's University Belfast, the Harvard-Smithsonian Center for Astrophysics, the Las Cumbres Observatory Global Telescope Network Incorporated, the National Central University of Taiwan, the Space Telescope Science Institute, the National Aeronautics and Space Administration under Grant No. NNX08AR22G issued through the Planetary Science Division of the NASA Science Mission Directorate, the National Science Foundation Grant No. AST-1238877, the University of Maryland, Eotvos Lorand University (ELTE), the Los Alamos National Laboratory, and the Gordon and Betty Moore Foundation.

Cao, H.-M., Frey, S., Gurvits, L. I., Yang, J., Hong, X.-Y., Paragi, Z., et al. (2014). VLBI observations of the radio quasar J2228+0110 at $z=5.95$ and other field sources in multiple-phase-centre mode. Astron. Astrophys. 563:A111. doi: 10.1051/0004-6361/201323328

Cao, H.-M., Frey, S., Gabányi, K. É, Paragi, Z., Yang, J., Cseh, D., et al. (2017). VLBI observations of four radio quasars at $z>4$ : blazars or not? Mon. Not. Roy. Astron. Soc. 467:950. doi: 10.1093/mnras/stx160

Chambers, K. C., Magnier, E. A., Metcalfe, N., Flewelling, H. A., Huber, M. E., Waters, C. Z., et al. (2016). The Pan-STARRS1 Surveys. ArXiv eprint: 1612.05560

Cheung, C. C., Stawarz, Ł., Siemiginowska, A., Gobeille, D., Wardle, J. F. C., Harris, D. E., et al. (2012). Discovery of a Kiloparsec-scale X-Ray/Radio Jet in the $z=4.72$ Quasar GB 1428+4217. Astrophys. J. 756:L20. doi: 10.1088/2041-8205/756/1/L20

Condon, J. J., Cotton, W. D., Greisen, E. W., Yin, Q. F., Perley, R. A., Taylor, G. B., et al. (1998). The NRAO VLA Sky Survey. Astron. J. 115:1693. doi: $10.1086 / 300337$

Constantin, A., Green, P., Aldcroft, T., Kim, D.-W., Haggard, D., Barkhouse, W., et al. (2009). Probing the balance of AGN and star-forming activity in the local Universe with ChaMP. Astrophys. J. 705:1336. doi: 10.1088/0004-637X/705/2/1336 
Coppejans, R., Frey, S., Cseh, D., Müller, C., Paragi, Z., Falcke, H., et al. (2016). On the nature of bright compact radio sources at $z>4.5$. Mon. Not. Roy. Astron. Soc. 463:3260. doi: 10.1093/mnras/stw2236

Eisenstein, D. J., Weinberg, D. H., Agol, E., Aihara, H., Allende Prieto, C., Anderson, S. F., et al. (2011). SDSS-III: massive spectroscopic surveys of the distant Universe, the Milky Way, and Extra-Solar Planetary Systems. Astron. J. 142:72. doi: 10.1088/0004-6256/142/3/72

Fan, X., Hennawi, J. F., Richards, G. T., Strauss, M. A., Schneider, D. P., Donley, J. L., et al. (2004). A Survey of $z>5.7$ Quasars in the Sloan Digital Sky Survey. III. Discovery of Five Additional Quasars. Astron. J. 128:515. doi: 10.1086/422434

Fey, A. L., Ma, C., Arias, E. F., Charlot, P. Feissel-Vernier, M., Gontier, A.-M., et al. (2004). The second extension of the international celestial reference frame: ICRF-EXT.1. Astron. J. 127:3587. doi: 10.1086/420998

Flesch, E. W. (2015). The Half Million Quasars (HMQ) Catalogue. PASA 32:10. doi: $10.1017 /$ pasa.2015.10

Frey, S., Mosoni, L., Paragi, Z., and Gurvits, L. I. (2003). Radio structure of the most distant radio-detected quasar at the 10-mas scale. Mon. Not. Roy. Astron. Soc. 343:L20. doi: 10.1046/j.1365-8711.2003.06869.x

Frey, S., Paragi, Z., Mosoni, L., and Gurvits, L. I. (2005). High-resolution radio imaging of the most distant radio quasar SDSS J0836+0054. Astron. Astrophys. 436:L13. doi: 10.1051/0004-6361:200500112

Frey, S., Gurvits, L. I., Paragi, Z., and Gabányi, K. É. (2008). High-resolution double morphology of the most distant known radio quasar at $z=6.12$. Astron. Astrophys. 484:L39. doi: 10.1051/0004-6361:200810040

Frey, S., Paragi, Z., Gurvits, L. I., Cseh, D., and Gabányi, K. É. (2010). Highresolution images of five radio quasars at early cosmological epochs. Astron. Astrophys. 524:A83. doi: 10.1051/0004-6361/201015554

Frey, S., Paragi, Z., Gurvits, L. I., Gabányi, K. É., and Cseh, D. (2011). Into the central $10 \mathrm{pc}$ of the most distant known radio quasar. VLBI imaging observations of J1429+5447 at $z=6.21$. Astron. Astrophys. 531:L5. doi: 10.1051/0004-6361/201117341

Frey, S., Fogasy, J. O., Paragi, Z., and Gurvits, L. I. (2013). On the Doppler boosting in the compact radio jet of the distant blazar J1026+2542 at $z=5.3$. Mon. Not. Roy. Astron. Soc. 431:1314. doi: 10.1093/mnras/stt249

Frey, S., Paragi, Z., Fogasy, J. O., and Gurvits, L. I. (2015). The first estimate of radio jet proper motion at $z>5$. Mon. Not. Roy. Astron. Soc. 446:2921. doi: 10.1093/mnras/stu2294

Gabányi, K. É., Cseh, D., Frey, S., Paragi, Z., Gurvits, L. I., An, T., et al. (2015). VLBI observation of the newly discovered $z=5.18$ quasar SDSS J0131-0321. Mon. Not. Roy. Astron. Soc. 450:L57. doi: 10.1093/mnrasl/slv046

Gurvits, L. I., Kellermann, K. I., and Frey, S. (1999). The "angular size - redshift" relation for compact radio structures in quasars and radio galaxies. Astron. Astrophys. 342:378.

Helmboldt, J. F., Taylor, G. B., Tremblay, S., Fassnacht, C. D., Walker, R. C., Myers, S. T., et al. (2007). The VLBA Imaging and Polarimetry Survey at $5 \mathrm{GHz}$. Astrophys. J. 658:203. doi: 10.1086/511005

Ivezić, Ž., Menou, K., Knapp, G. R., Strauss, M. A., Lupton, R. H., Vanden Berk, D. E. et al. (2002). Optical and radio properties of extragalactic sources observed by the FIRST survey and the Sloan Digital Sky Survey. Astron. J. 124:2364. doi: $10.1086 / 344069$

Jiang, L., McGreer, I. D., Fan, X., Strauss, M. A., Bañados, E., Becker, R. H., et al. (2016). The Final SDSS High-redshift Quasar Sample of 52 Quasars at $z>5.7$. Astrophys. J. 833:222. doi: 10.3847/1538-4357/833/2/222

Kalfountzou, E., Civano, F., Elvis, M., Trichas, M., and Green, P. (2014). The largest X-ray-selected sample of $\mathrm{z}>3$ AGNs: C-COSMOS and ChaMP. Mon. Not. Roy. Astron. Soc. 445:1430. doi: 10.1093/mnras/stu1745

Khorunzhev, G. A., Burenin, R. A., Sazonov, S. Y., Amvrosov, A. L., and Eselevich, M. V. (2017). Optical spectroscopy of candidates for quasars at $3<z<5.5$ from the XMM-newton X-ray survey. A distant X-ray quasar at $z=5.08$. Astr. L. 43:135. doi: 10.1134/S1063773717030045

Lynden-Bell, D. (1969). Galactic Nuclei as Collapsed Old Quasars. Nature 223:690. doi: $10.1038 / 223690 \mathrm{a} 0$

Marziani, P., and Sulentic, J. W. (2014). Highly accreting quasars: sample definition and possible cosmological implications. Mon. Not. Roy. Astron. Soc. 442:1211. doi: 10.1093/mnras/stu951

Matsuoka, Y., Onoue, M., Kashikawa, N., Iwasawa, K., Strauss, M. A., Nagao, T., et al. (2017). Subaru High-z Exploration of Low-Luminosity Quasars (SHELLQs).
II. Discovery of 32 Quasars and Luminous Galaxies at $5.7<z<6.8$. arXiv eprint:1704.05854

McMahon, R. G., White, R. L., Helfand, D. J., and Becker, R. H. (2002). Optical Counterparts for 70,000 Radio Sources: APM Identifications for the FIRST Radio Survey. Astrophys. J. 143:1. doi: 10.1086/342623

Momjian, E., Petric, A. O., and Carilli, C. L. (2004). VLBA Observations of $z>4$ Radio-loud Quasars. Astron. J. 127:587. doi: 10.1086/381300

Momjian, E., Carilli, C. L., and Petric, A. O. (2005). Sensitive VLBI Observations of the $z=4.7$ QSO BRI 1202-0725. Astron. J. 129:1809. doi: 10.1086/428598

Momjian, E., Carilli, C. L., and McGreer, I. D. (2008). Very Large Array and Very Long Baseline Array observations of the highest redshift radio-loud QSO J1427+3312 at $z=6.12$. Astron. J. 136:344. doi: 10.1088/0004-6256/136/1/344

Netzer, H. (2015). Revisiting the Unified Model of Active Galactic Nuclei. Annu. Rev. Astron. Astrophys. 53:365. doi: 10.1146/annurev-astro-082214-122302

Orsi, Á. A., Fanidakis, N., Lacey, C. G., and Baugh, C. M. (2016). The environments of high-redshift radio galaxies and quasars: probes of protoclusters. Mon. Not. Roy. Astron. Soc. 456:3827. doi: 10.1093/mnras/stv2919

Paragi, Z., Frey, S., Gurvits, L. I., Kellermann, K. I., Schilizzi, R. T., McMahon, R. G., et al. (1999). VLBI imaging of extremely high redshift quasars at $5 \mathrm{GHz}$. Astron. Astrophys. 344:51.

Parijskij, Y. N., Thomasson, P., Kopylov, A. I., Zhelenkova, O. P., Muxlow, T. W. B., Beswick, R., et al. (2014). Observations of the $z=4.514$ radio galaxy RC J0311+0507. Mon. Not. Roy. Astron. Soc. 439:2314. doi: 10.1093/mnras/stu047

Pâris, I., Petitjean, P., Ross, N. P., Myers, A. D., Aubourg, É., Streblyanska, A., et al. (2017). The Sloan Digital Sky Survey Quasar Catalog: Twelfth data release. Astron. Astrophys. 597:A25. doi: 10.1051/0004-6361/201527999

Petrov, L., Kovalev, Y. Y., Fomalont, E. B., and Gordon, D. (2006). The Fourth VLBA Calibrator Survey: VCS4. Astron. J. 131:1872. doi: 10.1086/499947

Petrov, L., Kovalev, Y. Y., Fomalont, E. B., and Gordon, D. (2008). The Sixth VLBA Calibrator Survey: VCS6. Astron. J. 136:580. doi: 10.1088/0004-6256/136/2/580

Reed, S. L., McMahon, R. G., Martini, P., Banerji, M., Auger, M., Hewett, P. C., et al. (2017). Eight new luminous $z>6$ quasars selected via SED model fitting of VISTA, WISE and Dark Energy Survey Year 1 Observations. arXiv eprint:1701.04852

Richards, G. T., Myers, A. D., Gray, A. G., Riegel, R. N., Nichol, R. C., Brunner, R. J., et al. (2009). Efficient photometric selection of quasars from the Sloan Digital Sky Survey. II. $\sim 1,000,000$ Quasars from Data Release 6. Astrophys. J. 180:67. doi: 10.1088/0067-0049/180/1/67

Rimoldini, L., Dubath, P., Süveges, M., López, M., Sarro, L. M., Blomme, J., et al. (2012). Automated classification of Hipparcos unsolved variables. Mon. Not. Roy. Astron. Soc. 427:2917. doi: 10.1111/j.1365-2966.2012.21752.x

Romani R. W., Sowards-Emmerd, D., Greenhill, L., and Michelson, P. (2004) Q0906+6930: The highest redshift blazar. Astrophys. J. 610:L9. doi: $10.1086 / 423201$

Sadler, E. M., Jackson, C. A., Cannon, R. D., McIntyre, V. J., Murphy, T., BlandHawthorn, J., et al. (2002). Radio sources in the 2dF Galaxy Redshift Survey - II. Local radio luminosity functions for AGN and star-forming galaxies at $1.4 \mathrm{GHz}$. Mon. Not. Roy. Astron. Soc. 329:227. doi: 10.1046/j.1365-8711.2002.04998.x

Secrest, N. J., Dudik, R. P., Dorland, B. N., Zacharias, N., Makarov, V., Fey, A., et al. (2015). Identification of 1.4 Million Active Galactic Nuclei in the Mid-Infrared using WISE Data. Astrophys. J. 221:12. doi: 10.1088/0067-0049/221/1/12

Shen, Y., Richards, G. T., Strauss, M. A., Hall, P. B., Schneider, D. P., Snedden, S., et al. (2011). A Catalog of Quasar Properties from Sloan Digital Sky Survey Data Release 7. Astrophys. J. 194:45. doi: 10.1088/0067-0049/194/2/45

Tang, J., Goto, T., Ohyama, Y., Chen, W.-P., Walter, F., Venemans, B., et al. (2017). A quasar discovered at redshift 6.6 from Pan-STARRS1. Mon. Not. Roy. Astron. Soc. 466:4568. doi: 10.1093/mnras/stw3287

Taylor, M. B. (2005). “TOPCAT and STIL: Starlink Table/VOTable Processing Software," in Astronomical Data Analysis Software and Systems, XIV ASP Conference Series (Pasadena), 347:29.

Tuccillo, D., Bruni, G., DiPompeo, M. A., Brotherton, M. S., Pasetto, A., Kraus, A., et al. (2017). A multiwavelength continuum characterization of highredshift broad absorption line quasars. Mon. Not. Roy. Astron. Soc. 467:4763. doi: 10.1093/mnras/stx333

Uchiyama, H., Toshikawa, J., Kashikawa, N., Overzier, R., Chiang, Y.-K., Tanaka, M., et al. (2017). Luminous quasars do not live in the most overdense regions of Galaxies at $z \sim 4$. arXiv eprint:1704.06050 
Urry, C. M., and Padovani, P. (1995). Unified Schemes for Radio-Loud Active Galactic Nuclei. PASP 107:803. doi: 10.1086/133630

Veres, P., Frey, S. Paragi, Z., and Gurvits, L. I. (2010). Physical parameters of a relativistic jet at very high redshift: the case of the blazar J1430+4204. Astron. Astrophys. 521:A6. doi: 10.1051/0004-6361/201014957

Véron-Cetty, M. P., and Véron, P. (2010). A catalogue of quasars and active nuclei: 13th edition. Astron. Astrophys. 518A:10. doi: 10.1051/0004-6361/201014188

Wang, F., Wu, X., Fan, X., Yang, J., Yi, W., Bian, F., et al. (2016). A Survey of Luminous High-redshift Quasars with SDSS and WISE. I. Target Selection and Optical Spectroscopy. Astrophys. J. 819:24. doi: 10.3847/0004-637X/819/1/24

Wang, F., Fan, X., Yang, J., Wu, X.-B., Yang, Q., Bian, F., et al. (2017). First Discoveries of $z>6$ Quasars with the DECam Legacy Survey and UKIRT Hemisphere Survey. Astrophys. J. 839:27. doi: 10.3847/1538-4357/aa689f

Wang, R., Momjian, E., Carilli, C. L., Wu, X.-B., Fan, X., Walter, F., et al. (2017). Milliarcsecond Imaging of the Radio Emission from the Quasar with the Most Massive Black Hole at Reionization. Astrophys. J. 835:L20. doi: 10.3847/2041-8213/835/2/L20

Warren, S. J., Hewett, P. C., Irwin, M. J., McMahon, R. G., Bridgeland, M. T., Bunclark, P. S., et al. (1987). First observation of a quasar with a redshift of 4. Nature 325:131. doi: 10.1038/325131a0

White, R. L., Becker, R. H., Helfand, D. J., and Gregg, M. D. (1997). A Catalog of $1.4 \mathrm{GHz}$ Radio Sources from the FIRST Survey. Astrophys. J. 475:479. doi: $10.1086 / 303564$

Willott, C. J., Delorme, P., Reylé, C., Albert, L., Bergeron, J., Crampton, D., et al. (2010). The Canada-France High-z Quasar Survey: Nine New Quasars and the Luminosity Function at Redshift 6. Astron. J. 139:906. doi: 10.1088/0004-6256/139/3/906
Yang, J., Fan, X., Wu, X., Wang, Fe., Bian, F., Yang, Q., et al. (2017). Discovery of 16 New $z \sim 5.5$ Quasars: Filling in the Redshift Gap of Quasar Color Selection. Astron. J. 153:184. doi: 10.3847/1538-3881/aa6577

Yi, W.-M., Wang, F., Wu, X.-B., Yang, J., Bai, J.-M., Fan, X., et al. (2014). SDSS J013127.34-032100.1: A Newly Discovered Radio-loud Quasar at $z=5.18$ with Extremely High Luminosity. Astrophys. J. 795:L29. doi: 10.1088/2041-8205/795/2/L29

Yi, W., Green, R., Bai, J.-M., Wang, T., Grier, C. J., Trump, J. R., et al. (2017). The Physical Constraints on a New LoBAL QSO at $z=4.82$. Astrophys. J. 838:135. doi: 10.3847/1538-4357/aa65d6

York, D. G., Adelman, J., Anderson, J. E. Jr., Anderson, S. F., Annis, J., Bahcall, N. A., et al. (2000). The Sloan Digital Sky Survey: technical summary. Astron. J. 120:1579. doi: $10.1086 / 301513$

Zhang, Y., An, T., Frey, S., Gabányi, K. É., Paragi, Z., Gurvits, L. I., et al. (2017). J0906+6930: a radio-loud quasar in the early Universe. Mon. Not. Roy. Astron. Soc. 468:69. doi: 10.1093/mnras/stx392

Conflict of Interest Statement: The authors declare that the research was conducted in the absence of any commercial or financial relationships that could be construed as a potential conflict of interest.

Copyright (c) 2017 Perger, Frey, Gabányi and Tóth. This is an open-access article distributed under the terms of the Creative Commons Attribution License (CC BY). The use, distribution or reproduction in other forums is permitted, provided the original author(s) or licensor are credited and that the original publication in this journal is cited, in accordance with accepted academic practice. No use, distribution or reproduction is permitted which does not comply with these terms. 